\title{
Quantum Teleportation of an Arbitrary N-qubit State via EPR Channels Bo ZHANG ${ }^{1, a,{ }^{*}}$, Xingtong LIU, Jian WANG, Chaojing Tang \\ ${ }^{1}$ School of Electronic Science and Engineering, National University of Defense Technology, Changsha, 410073, China \\ aemail: yumingsec@sina.com, ${ }^{*}$ corresponding author
}

Keywords: Quantum Teleportation; EPR Channel; Arbitrary $N$-qubit State; Mathematical Induction

\begin{abstract}
This paper demonstrates that an arbitrary $N$-qubit state can be faithfully and deterministically teleported from Alice to Bob via $N$ pairs of EPR channels. The analytical expression of the reconstruct criterion is derived explicitly in the most general case, with a strict proof through mathematical induction method.
\end{abstract}

\section{Introduction}

Quantum teleportation is a demonstration of the astonishing features of quantum mechanics, which can transport an unknown quantum state from one site to another via previously shared entanglement, classical communications and local operations. In 1993, Bennett et al. [1] proposed the first quantum teleportation protocol, using a maximally entangled two-qubit state to teleport an arbitrary single-qubit state. In 1997, this protocol was experimentally demonstrated [2]. Thereafter, perfect teleportation of an arbitrary single-qubit state, using tripartite GHZ state [3], fourpartite GHZ state [4], SLOCC equivalent W-class state [5] and cluster state [6] were proposed, respectively. Meanwhile, perfect teleportation of an arbitrary two-qubit state, using tensor product of two Bell states [7], tensor product of two orthogonal states [8], genuinely entangled five-qubit state [9], five-qubit cluster state [10] and six-qubit genuinely entangled state [11] were proposed, respectively. When it comes to $\mathrm{N}$-qubit states, there are schemes that can only teleport certain kinds of states, such as $\mathrm{N}$-qubit state of generalized Bell-type[12], N-qubit W state[13], N-qubit W-like state[14] and N-qubit GHZ state[15]. There are also research on the teleportation of an arbitrary $\mathrm{N}$-qubit state employing various channels, e.g., non-maximally entangled Bell state channel [16], the composite GHZ-Bell channel [17], genuine multipartite entanglement quantum channel [18, 19] and $\mathrm{N}$ pairs of EPR channel [7, 20]. In [16], the scheme succeeds with unit fidelity but less than unit probability. All the schemes in [7, 17-20] can accomplish the teleportation deterministically.

However, in [17-19], multi-particle joint measurement is required and no analytical expression of the criterion is provided. In [7], $\mathrm{N}$ Bell state measurements are required but no proof was provided. In [20], Latin square was used to deduce the criterion, which is not a strict proof for arbitrary $\mathrm{N}$ either. This motivates us to further study the teleportaion of an arbitrary $\mathrm{N}$-qubit state via EPR channels to provide an explicit analytical expression of the reconstruct criterion as well as a strict proof.

In this paper, we explicitly show that an arbitrary $\mathrm{N}$-qubit state can be faithfully and deterministically teleported from Alice to Bob via N pairs of EPR channels. It only requires N Bell state measurements by the sender and $\mathrm{N}$ single-qubit transformations by the receiver. The strict proof through mathematical induction is presented and the analytical expression of the criterion for the receiver to reconstruct the desired state is derived explicitly in the most general case.

\section{Quantum Teleportation of an Arbitrary N-qubit State}

The most general form of a $N$-qubit state is given by

$$
|\phi\rangle_{x_{1} x_{2} \ldots x_{N}}=\sum_{j=0}^{2^{N}-1} \alpha_{j}\left|y_{j}\right\rangle_{x_{1} x_{2} \ldots x_{N}},
$$

where $\alpha_{j}$ are complex coefficients, $y_{j}$ is the binary representation of the number $j$ and we 
assume $|\phi\rangle_{x_{1} x_{2} \ldots x_{N}}$ to be normalized. A set of EPR states are given by

$$
\begin{aligned}
\left|G_{00}\right\rangle & =\left|\eta^{+}\right\rangle=\frac{1}{\sqrt{2}}(|00\rangle+|11\rangle) \\
\left|G_{01}\right\rangle & =\left|\eta^{-}\right\rangle=\frac{1}{\sqrt{2}}(|00\rangle-|11\rangle) \\
\left|G_{10}\right\rangle & =\left|\psi^{+}\right\rangle=\frac{1}{\sqrt{2}}(|01\rangle+|10\rangle) \\
\left|G_{11}\right\rangle & =\left|\psi^{-}\right\rangle=\frac{1}{\sqrt{2}}(|01\rangle-|10\rangle) .
\end{aligned}
$$

Suppose Alice has an unknown $N$-qubit state, $|\phi\rangle_{x_{1} x_{2} \ldots x_{N}}$, which is shown in Eq. (1). She wants to send this state to a distant receiver Bob. Alice and Bob share $N$ EPR states in advance, say

$$
\left|\eta^{+}\right\rangle_{12} \otimes\left|\eta^{+}\right\rangle_{34} \otimes \ldots \otimes\left|\eta^{+}\right\rangle_{(2 N-1)(2 N)}=\otimes_{k=1}^{N}\left|\eta^{+}\right\rangle_{(2 k-1)(2 k)},
$$

with the qubits $x_{1}, x_{2} \ldots x_{N}, 1,3,5, \ldots 2 N-1$ belong to Alice, while qubits $2,4 \ldots 2 N$ belong to Bob, respectively.

The combined state of the whole $3 N$-qubit system is given by,

$$
\begin{aligned}
& |\Gamma\rangle_{x_{1} x_{2} \ldots x_{N} 123456 \ldots(2 N-1)(2 N)} \\
= & |\phi\rangle_{x_{1} x_{2} \ldots x_{N}} \otimes_{k=1}^{N}\left|\eta^{+}\right\rangle_{(2 k-1)(2 k)} \\
= & \frac{1}{2^{N}} \sum_{j=0}^{4^{N}-1}\left[\otimes_{k=1}^{N}\left|G_{j_{2 k-1} j_{2 k}}\right\rangle_{x_{k}(2 k-1)} \otimes\left|\phi_{j}\right\rangle_{24 \ldots(2 N)}\right],
\end{aligned}
$$

where $\left|\phi_{j}\right\rangle_{24 \ldots(2 N)}=U_{j}|\phi\rangle_{x_{1} x_{2} \ldots x_{N}}$ and $U_{j}=\otimes_{k=1}^{N}\left(\delta_{k}^{x}\right)^{j_{2 k-1}}\left(\delta_{k}^{z}\right)^{j_{2 k}} . j_{k}$ represents the $k_{t h}$ bit (from left to right) of the number $0 \leq j \leq 4^{N}-1$, which is written in binary notation and zeros should be added to leave all $j$ 's with the same number of bits (2N bits). The subindex $k$ indicates on which qubit the Pauli matrices $\delta^{x}$ and $\delta^{z}$ should operate. For consistency, the proof of Eq.(4) is presented in the next section.

Based on Eq. (4), the arbitrary $N$-qubit teleportation protocol is shown as follows.

(1) Alice shares $N$ sets of EPR states $\otimes_{k=1}^{N}\left|\eta^{+}\right\rangle_{(2 k-1)(2 k)}$ with Bob, with the qubits $x_{1}, x_{2}, \ldots x_{N}, 1,3$, $5 \ldots 2 \mathrm{~N}-1$ belong to Alice, while qubits $2,4 \ldots 2 \mathrm{~N}$ belong to Bob, respectively.

(2) Alice performs $N$ Bell state measurements on qubits $\left(x_{1}, 1\right), \ldots\left(x_{N}, 2 N-1\right)$ in the basis of $\left\{\left|G_{00}\right\rangle,\left|G_{01}\right\rangle,\left|G_{10}\right\rangle,\left|G_{11}\right\rangle\right\}$, respectively. Alice and Bob agree that the two classical bits $\{00,01,10,11\}$ represent the measurement outcome $\left\{\left|G_{00}\right\rangle,\left|G_{01}\right\rangle,\left|G_{10}\right\rangle,\left|G_{11}\right\rangle\right\}$, correspondingly. So after $N$ Bell state measurements on her qubits, Alice will obtain one of the $4^{N}$ states with equal probabilities, represented by an $2 N$ - bit classical message, $Y=\left(j_{1} j_{2}\right)\left(j_{3} j_{4}\right) \ldots\left(j_{2 N-1} j_{2 N}\right)$, where $j_{1} j_{2}, j_{3} j_{4}, \ldots, j_{2 N-1} j_{2 N}$ represent the measurement results of qubit pairs $\left(x_{1}, 1\right),\left(x_{2}, 3\right) \ldots,\left(x_{N}, 2 N-1\right)$, correspondingly.

(3) Alice sends $Y$ to Bob.

(4) Bob recovers his $N$ qubits to the original state $\left|\phi_{j}\right\rangle$ by performing unitary operations $U_{j}=\otimes_{k=1}^{N}\left(\delta_{k}^{x}\right)^{j_{2 k-1}}\left(\delta_{k}^{z}\right)^{j_{2 k}}$, which is an analytical expression of the reconstruct criterion instructed by $Y$. Again, the subindex $k$ indicates on which qubit the Pauli matrices $\delta^{x}$ and $\delta^{z}$ should operate, where $\delta^{x}=|1\rangle\langle 0|+| 0\rangle\langle 1|$ and $\delta^{z}=|0\rangle\langle 0|-| 1\rangle\langle 1|$.

Up to now, the teleportation is finished faithfully and deterministically, i.e., the success probability is $100 \%$. Let us illustrate the protocol with two concrete examples. For $N=2$, Alice's measurement result and Bob's corresponding operations are listed in Table 1, while for $N=3$, they are listed in Table 2.

\begin{tabular}{ll}
\hline Alice's result & Bob's operatioin \\
$\left|\eta^{ \pm}\right\rangle_{x_{1}} \otimes\left|\eta^{ \pm}\right\rangle_{x_{2} 3}$ & $(|0\rangle\langle 0| \pm| 1\rangle\langle 1|)_{2} \otimes(|0\rangle\langle 0| \pm| 1\rangle\langle 1|)_{4}$ \\
$\left|\eta^{ \pm}\right\rangle_{x_{1} 1} \otimes\left|\psi^{ \pm}\right\rangle_{x_{2} 3}$ & $(|0\rangle\langle 0| \pm| 1\rangle\langle 1|)_{2} \otimes(|1\rangle\langle 0| \pm| 0\rangle\langle 1|)_{4}$ \\
$\left|\psi^{ \pm}\right\rangle_{x_{1} 1} \otimes\left|\eta^{ \pm}\right\rangle_{x_{2} 3}$ & $(|1\rangle\langle 0| \pm| 0\rangle\langle 1|)_{2} \otimes(|0\rangle\langle 0| \pm| 1\rangle\langle 1|)_{4}$ \\
$\left|\psi^{ \pm}\right\rangle_{x_{1} 1} \otimes\left|\psi^{ \pm}\right\rangle_{x_{2} 3}$ & $(|1\rangle\langle 0| \pm| 0\rangle\langle 1|)_{2} \otimes(|1\rangle\langle 0| \pm| 0\rangle\langle 1|)_{4}$ \\
\hline
\end{tabular}

Table 1. Alice’s measurement result and Bob’s corresponding operations for $N=2$. 


\begin{tabular}{ll}
\hline Alice's result & Bob's operatioin \\
$\left|\eta^{ \pm}\right\rangle_{x_{1} 1} \otimes\left|\eta^{ \pm}\right\rangle_{x_{2} 3} \otimes\left|\eta^{ \pm}\right\rangle_{x_{3} 5}$ & $(|0\rangle\langle 0| \pm| 1\rangle\langle 1|)_{2} \otimes(|0\rangle\langle 0| \pm| 1\rangle\langle 1|)_{4} \otimes(|0\rangle\langle 0| \pm| 1\rangle\langle 1|)_{6}$ \\
$\left|\eta^{ \pm}\right\rangle_{x_{1} 1} \otimes\left|\eta^{ \pm}\right\rangle_{x_{2} 3} \otimes\left|\psi^{ \pm}\right\rangle_{x_{3} 5}$ & $(|0\rangle\langle 0| \pm| 1\rangle\langle 1|)_{2} \otimes(|0\rangle\langle 0| \pm| 1\rangle\langle 1|)_{4} \otimes(|1\rangle\langle 0| \pm| 0\rangle\langle 1|)_{6}$ \\
$\left|\eta^{ \pm}\right\rangle_{x_{1} 1} \otimes\left|\psi^{ \pm}\right\rangle_{x_{2} 3} \otimes\left|\eta^{ \pm}\right\rangle_{x_{3} 5}$ & $(|0\rangle\langle 0| \pm| 1\rangle\langle 1|)_{2} \otimes(|1\rangle\langle 0| \pm| 0\rangle\langle 1|)_{4} \otimes(|0\rangle\langle 0| \pm| 1\rangle\langle 1|)_{6}$ \\
$\left|\eta^{ \pm}\right\rangle_{x_{1} 1} \otimes\left|\psi^{ \pm}\right\rangle_{x_{2} 3} \otimes\left|\psi^{ \pm}\right\rangle_{x_{3} 5}$ & $(|0\rangle\langle 0| \pm| 1\rangle\langle 1|)_{2} \otimes(|1\rangle\langle 0| \pm| 0\rangle\langle 1|)_{4} \otimes(|1\rangle\langle 0| \pm| 0\rangle\langle 1|)_{6}$ \\
$\left|\psi^{ \pm}\right\rangle_{x_{1} 1} \otimes\left|\eta^{ \pm}\right\rangle_{x_{2} 3} \otimes\left|\eta^{ \pm}\right\rangle_{x_{3} 5}$ & $(|1\rangle\langle 0| \pm| 0\rangle\langle 1|)_{2} \otimes(|0\rangle\langle 0| \pm| 1\rangle\langle 1|)_{4} \otimes(|0\rangle\langle 0| \pm| 1\rangle\langle 1|)_{6}$ \\
$\left|\psi^{ \pm}\right\rangle_{x_{1} 1} \otimes\left|\eta^{ \pm}\right\rangle_{x_{2} 3} \otimes\left|\psi^{ \pm}\right\rangle_{x_{3} 5}$ & $(|1\rangle\langle 0| \pm| 0\rangle\langle 1|)_{2} \otimes(|0\rangle\langle 0| \pm| 1\rangle\langle 1|)_{4} \otimes(|1\rangle\langle 0| \pm| 0\rangle\langle 1|)_{6}$ \\
$\left|\psi^{ \pm}\right\rangle_{x_{1} 1} \otimes\left|\psi^{ \pm}\right\rangle_{x_{2} 3} \otimes\left|\eta^{ \pm}\right\rangle_{x_{3} 5}$ & $(|1\rangle\langle 0| \pm| 0\rangle\langle 1|)_{2} \otimes(|1\rangle\langle 0| \pm| 0\rangle\langle 1|)_{4} \otimes(|0\rangle\langle 0| \pm| 1\rangle\langle 1|)_{6}$ \\
$\left|\psi^{ \pm}\right\rangle_{x_{1} 1} \otimes\left|\psi^{ \pm}\right\rangle_{x_{2} 3} \otimes\left|\psi^{ \pm}\right\rangle_{x_{3} 5}$ & $(|1\rangle\langle 0| \pm| 0\rangle\langle 1|)_{2} \otimes(|1\rangle\langle 0| \pm| 0\rangle\langle 1|)_{4} \otimes(|1\rangle\langle 0| \pm| 0\rangle\langle 1|)_{6}$ \\
\hline
\end{tabular}

Table 2. Alice’s measurement result and Bob’s corresponding operations for $N=3$.

\section{Proof}

Eq. (4) is proved through mathematical induction. Now, apply induction on $N$ to Eq. (4).

Step 1. Verify that Eq. (4) holds for $\mathrm{N}=1$.

When $N=1,|\phi\rangle_{x_{1}}=\alpha_{0}|0\rangle_{x_{1}}+\alpha_{1}|1\rangle_{x_{1}}$. The combined state of the 3-qubit system is given by $|\Gamma\rangle_{x_{1} 12}=|\phi\rangle_{x_{1}} \otimes\left|\eta^{+}\right\rangle_{12}$

$$
\begin{aligned}
& =\left(\alpha_{0}|0\rangle_{x_{1}}+\alpha_{1}|1\rangle_{x_{1}}\right) \otimes \frac{1}{\sqrt{2}}(|00\rangle+|11\rangle)_{12} \\
& =\frac{1}{2}\left[\left|\eta^{+}\right\rangle_{x_{1} 1}\left(\alpha_{0}|0\rangle_{2}+\alpha_{1}|1\rangle_{2}\right)+\left|\eta^{-}\right\rangle_{x_{1} 1}\left(\alpha_{0}|0\rangle_{2}-\alpha_{1}|1\rangle_{2}\right)\right. \\
& \left.+\left|\psi^{+}\right\rangle_{x_{1} 1}\left(\alpha_{1}|0\rangle_{2}+\alpha_{0}|1\rangle_{2}\right)+\left|\psi^{-}\right\rangle_{x_{1} 1}\left(\alpha_{1}|0\rangle_{2}-\alpha_{0}|1\rangle_{2}\right)\right] \\
& =\frac{1}{2}\left[\left|G_{00}\right\rangle_{x_{1} 1}(I|\phi\rangle)_{2}+\left|G_{01}\right\rangle_{x_{1} 1}\left(\delta^{z}|\phi\rangle\right)_{2}+\left|G_{10}\right\rangle_{x_{1} 1}\left(\delta^{x}|\phi\rangle\right)_{2}+\left|G_{11}\right\rangle_{x_{1} 1}\left(\delta^{x} \delta^{z}|\phi\rangle\right)_{2}\right] \\
& =\frac{1}{2} \sum_{j=0}^{3}\left[\left|G_{j_{1} j_{2}}\right\rangle_{x_{1} 1} \otimes\left(\left(\delta^{x}\right)^{j_{1}}\left(\delta^{z}\right)^{j_{2}}|\phi\rangle\right)_{2}\right] \\
& =\frac{1}{2} \sum_{j=0}^{3}\left[\left|G_{j_{1} j_{2}}\right\rangle_{x_{1} 1} \otimes\left|\phi_{j}\right\rangle_{2}\right]
\end{aligned}
$$

So Eq. (4) holds for $N=1$.

Step 2. Suppose that Eq. (4) holds for arbitray positive interger $m>1$, which means

$|\phi\rangle_{x_{1} x_{2} \ldots x_{m}} \otimes_{k=1}^{m}\left|\eta^{+}\right\rangle_{(2 k-1)(2 k)}=\frac{1}{2^{m}} \sum_{j=0}^{4^{m}-1}\left[\otimes_{k=1}^{m}\left|G_{j_{2 k-1} j_{2 k}}\right\rangle_{x_{k}(2 k-1)} \otimes\left|\phi_{j}\right\rangle_{24 \ldots(2 m)}\right]$,

verify that Eq. (4) still holds for $\mathrm{N}=\mathrm{m}+1$.

Note that an arbitrary $(m+1)$-qubit state is given by

$$
\begin{aligned}
|\phi\rangle_{x_{1} x_{2} \ldots x_{m+1}} & =\sum_{j=0}^{2^{m+1}-1} \alpha_{j}\left|y_{j}\right\rangle_{x_{1} x_{2} \ldots x_{m+1}} \\
& =\sum_{j=0}^{2^{m}-1} \alpha_{j}\left|y_{j}\right\rangle_{x_{1} x_{2} \ldots x_{m+1}}+\sum_{j=2^{m}}^{2^{m+1}-1} \alpha_{j}\left|y_{j}\right\rangle_{x_{1} x_{2} \ldots x_{m+1}} \\
& =|0\rangle_{x_{1}} \otimes \sum_{j=0}^{2^{m}-1} \alpha_{j}\left|y_{j}\right\rangle_{x_{2} \ldots x_{m+1}}+|1\rangle_{x_{1}} \otimes \sum_{j=2^{m}}^{2^{m+1}-1} \alpha_{j}\left|y_{j}\right\rangle_{x_{2} \ldots x_{m+1}} \\
& =|0\rangle_{x_{1}} \otimes \sum_{j=0}^{2^{m}-1} \alpha_{j}\left|y_{j}\right\rangle_{x_{2} \ldots x_{m+1}}+|1\rangle_{x_{1}} \otimes \sum_{j=0}^{2^{m}-1} \beta_{j}\left|y_{j}\right\rangle_{x_{2} \ldots x_{m+1}},
\end{aligned}
$$

where $\quad \beta_{j}=\alpha_{j+2^{m}}$.

When $N=m+1$, based on Eq. (6) and Eq. (7),the combined state of the $3(m+1)$-qubit system is given by 


$$
\begin{array}{ll} 
& |\Gamma\rangle_{x_{1} x_{2} \ldots x_{m+1} 12 \ldots(2 m+1)(2 m+2)} \\
=\quad & |\phi\rangle_{x_{1} x_{2} \ldots x_{m+1}} \otimes_{k=1}^{m+1}\left|\eta^{+}\right\rangle_{(2 k-1)(2 k)} \\
=\quad & |0\rangle_{x_{1}} \otimes\left|\eta^{+}\right\rangle_{12} \otimes\left(\sum_{j=0}^{2^{m}-1}\left|\alpha_{j}\right|^{2}\right)^{\frac{1}{2}}\left(\sum_{j=0}^{2^{m}-1}\left|\alpha_{j}\right|^{2}\right)^{-\frac{1}{2}} \sum_{j=0}^{2^{m}-1} \alpha_{j}\left|y_{j}\right\rangle_{x_{2} \ldots x_{m+1}} \otimes_{k=2}^{m+1}\left|\eta^{+}\right\rangle_{(2 k-1)(2 k)} \\
& +|1\rangle_{x_{1}} \otimes\left|\eta^{+}\right\rangle_{12} \otimes\left(\sum_{j=0}^{2^{m}-1}\left|\beta_{j}\right|^{2}\right)^{\frac{1}{2}}\left(\sum_{j=0}^{2^{m}-1}\left|\beta_{j}\right|^{2}\right)^{-\frac{1}{2}} \sum_{j=0}^{2^{m}-1} \beta_{j}\left|y_{j}\right\rangle_{x_{2} \ldots x_{m+1}} \otimes_{k=2}^{m+1}\left|\eta^{+}\right\rangle_{(2 k-1)(2 k)} \\
=\quad & |0\rangle_{x_{1}} \otimes\left|\eta^{+}\right\rangle_{12} \otimes \frac{1}{2^{m}} \sum_{j=0}^{4^{m}-1}\left[\otimes_{k=1}^{m}\left|G_{j_{2 k-1} j_{2 k}}\right\rangle_{x_{k+1}(2 k+1)} \otimes\left|\phi_{j}\right\rangle_{46 \ldots(2 m+2)}\right] \\
& +|1\rangle_{x_{1}} \otimes\left|\eta^{+}\right\rangle_{12} \otimes \frac{1}{2^{m}} \sum_{j=0}^{4^{m}-1}\left[\otimes_{k=1}^{m}\left|G_{j_{2 k-1} j_{2 k}}\right\rangle_{x_{k+1}(2 k+1)} \otimes\left|\phi_{j}^{\prime}\right\rangle_{46 \ldots(2 m+2)}\right] .
\end{array}
$$

In Eq. (8), $\left|\phi_{j}\right\rangle_{46 \ldots(2 m+2)}=Q_{j} \sum_{j=0}^{2^{m}-1} \alpha_{j}\left|y_{j}\right\rangle_{x_{2} \ldots x_{m+1}},\left|\phi_{j}^{\prime}\right\rangle_{46 \ldots(2 m+2)}=Q_{j} \sum_{j=0}^{2^{m}-1} \beta_{j}\left|y_{j}\right\rangle_{x_{2} \ldots x_{m+1}}$, and $Q_{j}=\otimes_{k=1}^{m}\left(\delta_{k}^{x}\right)^{j_{2 k-1}}\left(\delta_{k}^{z}\right)^{j_{2 k}}$

Note that

$$
\begin{aligned}
|0\rangle_{x_{1}} \otimes\left|\eta^{+}\right\rangle_{12} & =\frac{1}{2}\left[\left(\left|\eta^{+}\right\rangle+\left|\eta^{-}\right\rangle\right)_{x_{1} 1}|0\rangle_{2}+\left(\left|\psi^{+}\right\rangle-\left|\psi^{-}\right\rangle\right)_{x_{1} 1}|1\rangle_{2}\right], \\
|1\rangle_{x_{1}} \otimes\left|\eta^{+}\right\rangle_{12} & =\frac{1}{2}\left[\left(\left|\psi^{+}\right\rangle+\left|\psi^{-}\right\rangle\right)_{x_{1} 1}|0\rangle_{2}+\left(\left|\eta^{+}\right\rangle-\left|\eta^{-}\right\rangle\right)_{x_{1} 1}|1\rangle_{2}\right],
\end{aligned}
$$

and define $\Omega, \Omega^{\prime}$ by

$$
\begin{aligned}
& \Omega=\sum_{j=0}^{4^{m}-1}\left[\otimes_{k=1}^{m}\left|G_{j_{2 k-1} j_{2 k}}\right\rangle_{x_{k+1}(2 k+1)} \otimes\left|\phi_{j}\right\rangle_{46 \ldots(2 m+2)}\right], \\
& \Omega^{\prime}=\sum_{j=0}^{4^{m}-1}\left[\otimes_{k=1}^{m}\left|G_{j_{2 k-1} j_{2 k}}\right\rangle_{x_{k+1}(2 k+1)} \otimes\left|\phi_{j}^{\prime}\right\rangle_{46 \ldots(2 m+2)}\right] .
\end{aligned}
$$

Combining Eq. (8) with Eq.(9) and (10),

$|\Gamma\rangle_{x_{1} x_{2} \ldots x_{m+1} 12 \ldots(2 m+1)(2 m+2)}$

$=\frac{1}{2^{m+1}}\left|\eta^{+}\right\rangle_{x_{1} 1}\left[|0\rangle_{2} \otimes \Omega+|1\rangle_{2} \otimes \Omega^{\prime}\right]+\frac{1}{2^{m+1}}\left|\eta^{-}\right\rangle_{x_{1} 1}\left[|0\rangle_{2} \otimes \Omega-|1\rangle_{2} \otimes \Omega^{\prime}\right]$

$+\quad \frac{1}{2^{m+1}}\left|\psi^{+}\right\rangle_{x_{1} 1}\left[|1\rangle_{2} \otimes \Omega+|0\rangle_{2} \otimes \Omega^{\prime}\right]+\frac{1}{2^{m+1}}\left|\psi^{-}\right\rangle_{x_{1} 1}\left[-|1\rangle_{2} \otimes \Omega+|0\rangle_{2} \otimes \Omega^{\prime}\right]$.

Note that

$$
\begin{aligned}
& R_{j}=\otimes_{k=2}^{m+1}\left(\delta_{k}^{x}\right)^{j_{2 k-3}}\left(\delta_{k}^{z}\right)^{j_{2 k-2}}, \\
& |0\rangle_{2}\left|\phi_{j}\right\rangle_{46 \ldots(2 m+2)}+|1\rangle_{2}\left|\phi_{j}^{\prime}\right\rangle_{46 \ldots(2 m+2)}=I_{1} \otimes R_{j}|\phi\rangle_{x_{1} x_{2} \ldots x_{m+1}}, \\
& |0\rangle_{2}\left|\phi_{j}\right\rangle_{46 \ldots(2 m+2)}-|1\rangle_{2}\left|\phi_{j}^{\prime}\right\rangle_{46 \ldots(2 m+2)}=\delta_{1}^{z} \otimes R_{j}|\phi\rangle_{x_{1} x_{2} \ldots x_{m+1}}, \\
& |1\rangle_{2}\left|\phi_{j}\right\rangle_{46 \ldots(2 m+2)}+|0\rangle_{2}\left|\phi_{j}^{\prime}\right\rangle_{46 \ldots(2 m+2)}=\delta_{1}^{x} \otimes R_{j}|\phi\rangle_{x_{1} x_{2} \ldots x_{m+1}}, \\
& -\quad|1\rangle_{2}\left|\phi_{j}\right\rangle_{46 \ldots(2 m+2)}+|0\rangle_{2}\left|\phi_{j}^{\prime}\right\rangle_{46 \ldots(2 m+2)}=\delta_{1}^{x} \delta_{1}^{z} \otimes R_{j}|\phi\rangle_{x_{1} x_{2} \ldots x_{m+1}} .
\end{aligned}
$$

Combining Eq. (11) with Eq. (12), 


$$
\begin{aligned}
& \quad|\Gamma\rangle_{x_{1} x_{2} \ldots x_{m+1} 12 \ldots(2 m+1)(2 m+2)} \\
& =\quad \frac{1}{2^{m+1}} \sum_{j=0}^{4^{m}-1}\left[\left|G_{00}\right\rangle_{x_{1} 1} \otimes_{k=1}^{m}\left|G_{j_{2 k-1} j_{2 k}}\right\rangle_{x_{k+1}(2 k+1)} \otimes I_{1} \otimes R_{j}|\phi\rangle_{x_{1} x_{2} \ldots x_{m+1}}\right] \\
& +\quad \frac{1}{2^{m+1}} \sum_{j=0}^{4^{m}-1}\left[\left|G_{01}\right\rangle_{x_{1} 1} \otimes_{k=1}^{m}\left|G_{j_{2 k-1} j_{2 k}}\right\rangle_{x_{k+1}(2 k+1)} \otimes \delta_{1}^{z} \otimes R_{j}|\phi\rangle_{x_{1} x_{2} \ldots x_{m+1}}\right] \\
& +\quad \frac{1}{2^{m+1}} \sum_{j=0}^{4^{m}-1}\left[\left|G_{10}\right\rangle_{x_{1} 1} \otimes_{k=1}^{m}\left|G_{j_{2 k-1} j_{2 k}}\right\rangle_{x_{k+1}(2 k+1)} \otimes \delta_{1}^{x} \otimes R_{j}|\phi\rangle_{x_{1} x_{2} \ldots x_{m+1}}\right] \\
& +\quad \frac{1}{2^{m+1}} \sum_{j=0}^{4^{m}-1}\left[\left|G_{11}\right\rangle_{x_{1} 1} \otimes_{k=1}^{m}\left|G_{j_{2 k-1} j_{2 k}}\right\rangle_{x_{k+1}(2 k+1)} \otimes \delta_{1}^{x} \delta_{1}^{z} \otimes R_{j}|\phi\rangle_{x_{1} x_{2} \ldots x_{m+1}}\right] \\
& =\frac{1}{2^{m+1}} \sum_{j=0}^{4^{m+1}-1}\left[\otimes_{k=1}^{m+1}\left|G_{j_{2 k-1} j_{2 k}}\right\rangle_{x_{k}(2 k-1)} \otimes\left|\phi_{j}\right\rangle_{24 \ldots(2 m+2)}\right] .
\end{aligned}
$$

That is, Eq. (4) still holds for $N=m+1$.

Up to now, we have strictly proved that Eq. (4) holds for arbitrary N, which guarantees the correctness of our protocol. Obviously, the proof itself is a derivation of the analytical expression of the criterion for the receiver to reconstruct the desired state.

\section{Conclusion}

We demonstrate that an arbitrary $N$-qubit state can be faithfully and deterministically teleported via N pairs of EPR channels. Only $N$ Bell state measurements by the sender and $N$ single-qubit transformations by the receiver are needed. The analytical expression of the reconstruct criterion is derived explicitly in the most general case, with a strict proof through mathematical induction method. Though the teleportation channel described in the protocol is $\mathrm{N}$ sets of $\left|\eta^{+}\right\rangle$, the results will be analogous for the channels formed by $\mathrm{N}$ sets of $\left|\eta^{-}\right\rangle,\left|\psi^{+}\right\rangle$or $\left|\psi^{-}\right\rangle$. Moreover, our proof method is universal, which can be applied in the teleportation of an arbitrary $N$-qubit state via other multi-particle entangled states, e.g., GHZ states, W states, etc.

\section{References}

[1] Bennett $\mathrm{C} \mathrm{H}$ and so on. Teleporting an unknown quantum state via dual classical and Einstein-Podolsky-Rosen channels. [J]. Physics Review Letter. 1993 (70) 1895-1899.

[2] Bouwmeester D, Pan J W, Mattle K, Eibl M, Weinfurter H, Zeilinger A. Experimental quantum teleportation. [J]. Nature 1997390 (6660).

[3] Karlsson A, Bourennane M. Quantum teleportation using three-particle entanglement. [J]. Physics Review A. 1998 (58) 4394-4400.

[4] Pati A K. Assisted cloning and orthogonal complementing of an unknown state. [J]. Physics Review A. 2000 (61) 022308.

[5] Agrawal P, Pati A. Perfect teleportation and superdense coding with W states. [J]. Physics Review A. 2006 (74) 062320.

[6] Briegel H J, Raussendorf R. Persistent entanglement in arrays of interacting particles. [J]. Physics Review Letter. 2001 (86) 910-913.

[7] Rigolin G. Quantum teleportation of an arbitrary two qubit state and its relation to multipartite entanglement. [J]. Physics Review A. 2005 (71) 032303.

[8] Yeo Y, Chua W K. Teleportation and dense coding with genuine multipartite entanglement. [J]. 
Physics Review Letter. 2006 (96) 060502.

[9] Muralidharan S, Panigrahi P K. Perfect teleportation, quantum-state sharing, and superdense coding through a genuinely entangled five-qubit state. [J]. Physics Review A. 2008 (77) 032321.

[10] Liu J C, Li Y H, Nie Y Y. Controlled teleportation of an arbitrary two-particle pure or mixed state by using a five-qubit cluster state. [J]. International Journal of Theoretical Physics. 49(8) 1976-1984.

[11] Li Y H, Liu J C, Nie Y Y. Quantum teleportation and quantum information splitting by using a genuinely entangled six-qubit state. [J]. International Journal of Theoretical Physics. 2010 49(10) 2592-2599.

[12] Liu Z M, Zhou L. Quantum teleportation of a three-qubit state using a five-qubit Cluster state. [J]. International Journal of Theoretical Physics. 2014 (53) 4079.

[13] Pathak A, Banerjee A. Effcient quantum circuits for perfect and controlled teleportation of n-qubit non-maximally entangled states of generalized bell-type. [J]. International Journal of Quantum Information. 2011 (09) 389-403.

[13] Zhong W X, Jun G, Guang-Ling C, Ai-Xi C. Teleportation of n-qubit W state without bell-state measurement via selective resonant interaction in cavity QED. [J]. Communications in Theoretical Physics. 2010 (54) 253-256.

[14] Man Z X, Xia Y J, An N. Quantum teleportation of an unknown n-qubit W-like state. [J]. JETP Letters. 2007 (85) 662-666.

[15] Jiang W X, Jian-Xing F, Shi-Qun Z, Jin-Qiao S. Controlled teleportation of an unknown n-qubit entangled GHZ state. [J]. Communications in Theoretical Physics. 2007 (47), 1045-1048.

[16] Liu Y L, Man Z X, Xia Y J. Quantum teleportation of an arbitrary n-qubit state via non-maximally entangled state. [J]. International Journal of Quantum Information. 2007 (05) 673-683.

[17] Saha D, Panigrahi P. N-qubit quantum teleportation, information splitting and superdense coding through the composite GHZ-bell channel. [J]. Quantum Information Processing. 2012 (11) 615-628.

[18] Chen P X, Zhu S Y, Guo G C. General form of genuine multipartite entanglement quantum channels for teleportation. [J]. Physics Review A. 2006 (74) 032324.

[19] Liu D M, Wang Y W, Jiang X M, Zheng Y Z. A criterion for quantum teleportation of an arbitrary N-particle state via a 2N-particle quantum channel. [J]. Chinese Physics B. 2010 (19) 020307.

[20] Cao M, Zhu S Q, Fang J X. Teleportation of n-particle state via n pairs of EPR channels. [J]. Communications in Theoretical Physics. 2004 (41) 689-692. 